\title{
Review of Hybrid Finite Element - Statistical Energy Analysis Methods in Vehicle NVH Predictions
}

\author{
M. F. Treszkai ${ }^{1}$, D. Feszty ${ }^{2}$ \\ Department of Whole Vehicle Development, Audi Hungaria Faculty of \\ Automotive Engineering, Széchenyi István University, \\ Egyetem tér 1., 9026 Györ, Hungary \\ e-mail ${ }^{1}$ : treszkai.marcell.ferenc@ga.sze.hu \\ e-mail²: feszty.daniel@sze.hu
}

Abstract: In the vehicle industry, the noise, vibration and harshness (NVH) characteristics of a car are becoming more and more important. In order to control these, it is necessary to know the sources and contributors to the interior noise level. This paper provide an overview of the state-ofthe-art on predicting the $\mathrm{NVH}$ characteristics of fully trimmed vehicles in the mid-frequency gap between $400 \mathrm{~Hz}$ to $1 \mathrm{kHz}$. It is shown that for this frequency range, typically a hybrid FE-SEA method is used. This method provides a mixture of the advantages of the deterministic and statistical approaches. The first part of the paper introduces the method, followed by an overview of the different fields of usage in the vehicle industry. At the end of the paper, gaps in the knowledge are identified.

Keywords: statistical energy analysis, hybrid finite element-statistical energy analysis, fully trimmed vehicle simulation

\section{Introduction}

Noise, Vibration and Harshness (NVH) research and development has become increasingly important in the vehicle industry recently. $\mathrm{NVH}$ is about predicting and controlling vibroacoustics phenomenon, which constitute a set of complex problems, such as structure-borne noise or airborne noise. Structure-borne noise can cause fatigue of car components as well as interior noise that is audible to the driver. The 
sources of noise or vibration occur at various frequency ranges, for example road noise occurs at lower frequency than wind induced noise.

There are also various trends in controlling the undesired vibroacoustics phenomenon. For example, the easiest way to reduce vibration or Sound Pressure Level (SPL) in a vehicle would be to add extra mass to a part participating in the vibration or noise transfer. However, this is problematic nowadays, when one of the main trends in the vehicle industry is to reduce the weight of the car due to the tightening emission standards, such as Euro 7. Also, electric vehicles have special importance from $\mathrm{NVH}$ point of view, since the excitation differs both in frequency and location in comparison to the classical internal combustion engine driven vehicles. The electric motor excitation occurs at a much higher frequency, than for combustion engines as well as noise sources, which have been "masked" before, might now become important. Such noise sources include road noise and wind induced noise. For the latter one, the turbulent boundary layer excitation will occur at higher frequencies and will be random. The heavy battery packages will increase the weight of an electric car, calling for the usage of new lightweight materials such as carbon fibre or alloys. At such complex or lightweight structures, one has to analyse the effect of high frequency excitation from structural, fatigue, failure as well as noise point of view. For this reason, electric vehicles require full car simulations, what represent a quite significant computational challenge [1].

From the frequency point of view, it is common to distinguish in vehicle $\mathrm{NVH}$ between low-frequency (up to $400 \mathrm{~Hz}$ ), mid-frequency $(400-1000 \mathrm{~Hz}$ ) and highfrequency (above $1000 \mathrm{~Hz}$ ) noise. There are quite matured methods established for predicting the low-frequency or high-frequency noise, however, there is no common approach to predict the mid-frequency problem. In this paper, the goal was to provide a comprehensive overview of the simulation methods for mid-frequency $\mathrm{NVH}$ problems, with a special focus on one of the most promising methods, the Hybrid Finite Element - Statistical Energy Analysis (Hybrid FE-SEA) method [2].

\section{Theoretical background of Hybrid FE-SEA}

In $\mathrm{NVH}$, the mid frequency range of excitations is defined as $\sim 400 \mathrm{~Hz}$ to $1 \mathrm{kHz}$ and in this region, the dynamic behaviour of a component becomes very important. At low frequencies, the modal density is low, the resonances are individual peaks, thus, it is quite easy to define the eigenmodes. However, when the frequency is higher, the modal density is also getting denser. Therefore, the modal behaviour of a component becomes more relevant, while the material inaccuracies and nonconstant thickness of a plate gets a higher importance [2]. However, FEM simulations are deterministic methods, and as such, they consider a perfect geometry. During comparison with measurements, one can observe that nominally 
identical geometries will yield a different result, raising the question: which one is the best?

In contrast, the Statistical Energy Analysis (SEA) method calculates with statistical background for the various material parameters, such as the density or the thickness. For this reason, SEA can represent more realistically the results of a set of measurements However, in the interest of high quality results, the method requires at least 3 modes per third octave band. This might be difficult to achieve for stiffer (such as beams) or smaller parts. Therefore, the philosophy of the Hybrid FE-SEA method is to combine the strengths of both methods, i.e. to use FEM for stiffer parts and SEA for plate-like structures [2].

\subsection{Finite Element Method}

The most common simulation method in vibroacoustics is the Finite Element Method (FEM), which provides good results up to $200-400 \mathrm{~Hz}$, depending on the complexity of the geometry. The problem is, however, that due to the deterministic nature of the method it is not applicable for mid- and high-frequencies, because small deviation in the material or manufacturing inaccuracies causes big differences in the results. In addition, the models require highly detailed geometries, as well as a quite fine mesh if the target frequency is high, since a finer mesh is required to capture a smaller wavelength of vibration. This leads to high total element number as well as computational costs. Since the lowest eigenmodes are the most important ones due to their high amplitude response, which can cause fatigue, these play great role in vibroacoustic analysis. However, Finite Element Method is still the best way to identify the eigenfrequencies and modeshapes, despite the fact that they neglect the uncertainty of manufacturing [2].

\subsection{Statistical Energy Analysis}

A common method for solving the high frequency domain is Statistical Energy Analyis (SEA). SEA is a statistical method, in which results are averaged spatially as well as throughout frequency. As a consequence, the method neglects most of the details of the model. The lower limit of the applicability of SEA method for vehicles is about $200-400 \mathrm{~Hz}$, because the SEA method needs a modal density of at least 3 modes per frequency band, which means a limitation at smaller subsystems or stiffer parts. In general, the frequency band is a third octave frequency band. Another issue in SEA is the identification of the Coupling Loss Factors (CLF). This value defines the energy loss at a junction, which of course would have an influence on the results. The Damping Loss Factor (DLF) - that is proportional with the subsystem structural damping - can be obtained in a different way, that also impact the results. The pure 
SEA is ideal for panel-like structures that have a large number of modal density [25].

The application of the SEA is widespread in the industry. Applications range from dishwashers and washing machines [2], to launch vehicles and spacecraft [3]. It has been well demonstrated to be used for predicting interior noise or for noise control treatment design in trains [6], ships [7], aircraft [8], buildings [9] and automotive applications [10], as well as for predicting the exterior noise for electric cars [1].

\subsubsection{Theoretical background}

The Statistical Energy Analysis was developed in the early 1960's when the vibrational response of a spacecraft launcher was to be predicted. The problem and the geometry were too complex to be solved via FEM. The number of modes of the Saturn launch vehicle was around 500000 in the frequency range from 0 to $2 \mathrm{kHz}$ [6]. Lyon [11] proposed that the energy flow between two coupled oscillators is proportional to the difference of the vibrational energies. The analogy of the two oscillators is similar to a heat transfer problem. Fig. 1 illustrates the latter one by considering two identical thermally conducting parts [5] [6].

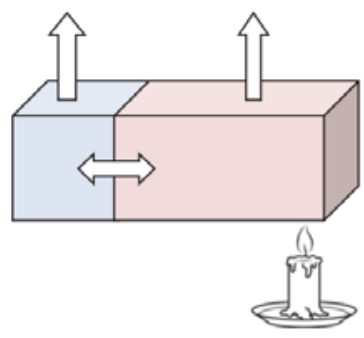

Figure 1. Heat flux analogy to the SEA [10]

According to Fig. 1, thermal energy is injected into the right subsystem, which one part dissipates to the environment, while the other part transfers heat energy into the left subsystem via the coupling. The modal density corresponds to the thermal capacity, the damping to radiation loss, the conductivity to the coupling loss factor and the flow of vibration energy as flow of heat [10].

The word "statistical" in the name of the method refers to the fact that the response is averaged over the subsystem as well as over a certain frequency band (generally $1 / 3$ octave). The energy, which is stored in the subsystem, dissipated or exchanged with other subsystems, is the main variable in the method. There are three approaches to calculate the transmission coefficient: a) modal, b) wave and c) mobility approach [4] [11]. 


\subsubsection{SEA assumptions}

Every numerical method has limitations, or is based on assumptions, which have to be taken into account in order to get reliable results in the simulations. The theory of SEA was derived by applying the following four assumptions [5] [12]:

- $\quad$ Rain-on-the-roof excitation

- $\quad$ Large number of modes

- Weak coupling

- $\quad$ Light damping

There are several publications, where the effect of these assumptions on the simulation results were investigated. Le Bot et al. [12] investigated in 2017 the effect of these assumptions on the results. The examinations were illustrated via simple examples. The first example consisted of 6 rectangular plates, with one plate excited by rain-on-the-roof random excitation, while the response was observed on one of the plates. Three calculations were performed: a reference calculation based on a closed equation, an SEA calculation and finally a geometrical acoustic prediction. Results showed that if the damping was light (about 1\%), then the SEA prediction was always correct. However, when the damping was strong (about 10\%), significant errors appeared. The second example consisted of three rectangular plates with random resonators and coupled through a spring of stiffness $K$. The coupling strength was controlled by varying $K$. Two calculations were performed: a) one with SEA, b) one by applying a semi-analytical method. The SEA solution always proved to be correct if the coupling was weak. However, after a certain point, the flow of the energy was reversed, giving negative values, what is physically impossible in thermodynamics. The conclusion of the article was that the first three assumptions can be reduced to a single condition of the diffuse field in all subsystems. The last assumption - weak coupling - is an imperative requirement that cannot be maintained in general.

\subsubsection{Statistical Energy Analysis equations}

The fundamental idea of the SEA method is to divide the geometry into subsystems, where the equations are solved. At first, consider a single subsystem eg. a flat plate - into which power is injected via excitation. This subsystem will store some of the vibrational energy, while the rest will be dissipated (see Fig. 2). [4]. 


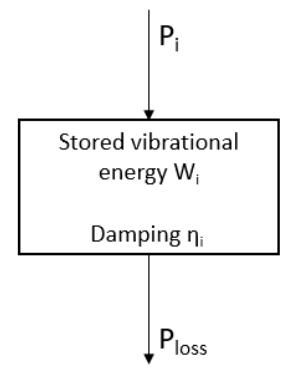

Figure 2. Power equilibirum of one subsystem [4]

The following equation describes this phenomenon [4] [13]:

$$
P_{i i}=\omega * \eta_{i} * W_{i}
$$

Where, $\mathrm{P}_{\mathrm{ii}}$ is the injected power, $\omega$ is circular frequency, $\eta_{i}$ is the damping loss factor and $\mathrm{W}_{\mathrm{i}}$ is the stored energy in the subsystem. In this case, the injected power equals the power loss, i.e. $P_{i i}=P_{\text {loss }}$.

In the case, where two subsystems are coupled to each other (see Fig. 3), the power balance equations will be [4] [13]:

$$
\left(\begin{array}{c}
P_{i} \\
P_{j}
\end{array}\right)=\omega\left(\begin{array}{cc}
\eta_{i}+\eta_{i j} & -\eta_{j i} \\
-\eta_{i j} & \eta_{j}+\eta_{j i}
\end{array}\right)\left(\begin{array}{c}
E_{i} \\
E_{j}
\end{array}\right)
$$

Note that the left side of the equation is the vector of input power, while the right side contains the frequency, the loss matrix, and the energy vector, respectively.

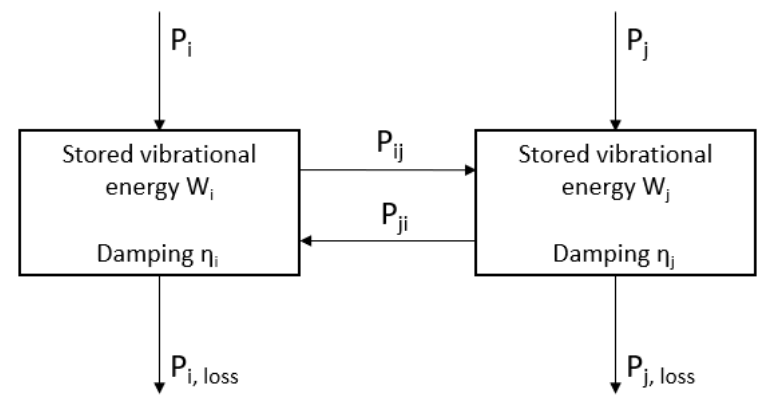

Figure 3. Power equilibrium of two coupled subsystems [4] 


\subsubsection{Energy of vibration}

In SEA, the main variable is the vibrational energy of a subsystem. There are two different subsystem types: structural and acoustic. The structural subsystem energy is given by the kinetic energy [4] [13]:

$$
E_{i}=m_{i} \cdot v_{i}^{2}
$$

Where $m_{i}$ is the weight of the subsystem, $v_{i}$ is the root-mean-square velocity, averaged over the subsystem.

The acoustic subsystem energy is [4] [13]:

$$
E_{i}=\frac{p_{i}^{2} V_{i}}{\rho_{i} c_{i}^{2}}
$$

Where, $p_{i}$ is the root-mean-square sound pressure level (SPL) in the acoustic cavity, marked as $V_{i}$. The continuum density is $\rho_{i}$ and the $c_{i}$ is the wave speed in the continuum.

\subsection{Hybrid FE-SEA}

The concept of the hybrid FE-SEA method is to combine the strengths of the two methods. Thus, some part of a subsystem is modelled in FE while the rest in SEA. The part modelled in FE has well defined physical properties, which values are equal over a part. On the other hand, the SEA subsystem has random physical parameters, such as density or geometry, etc. For this reason, the hybrid model contains enough random, or so-called statistical parameters, which makes the system response more realistic [2]. The general guideline for dividing the system is the following: stiffer parts are modelled in FE, since they have small modal density at higher frequencies (for example, the A-pillar of a vehicle). Larger panels, or plates with high modal density are, on the other hand, considered as SEA subsystems (for example, the roof or windows). The SEA subsystems are then coupled to the FEM systems [2][14]. The hybrid FE-SEA subsystem energy balance equations the following [2]:

$$
\omega\left(\eta_{j}+\eta_{d, j}\right) E_{j}+\sum_{k} \omega \eta_{j k} n_{j}\left(\frac{E_{j}}{n_{j}}-\frac{E_{k}}{n_{k}}\right)=P_{i n, j}^{e x t}+P_{i n, j}
$$

The equation based on the original SEA equation supplemented with the FEM equation. The $\eta_{j}$ is the damping loss factor of subsystem $j, n_{j}$ the modal density of subsystem $j, P_{i n, j}^{\text {ext }}$ the power input.

Different hybrid solutions are being, since both acoustic and structural components can be included in the model [15]. This is a quite new method that alloys the advantages of the two methods. The hybrid method main advantages the model 
size is smaller compare to the FEM model, and more suitable for the mid-frequency range. In addition, the hybrid model much faster for a fully trimmed vehicle then the pure FEM, and it give an opportunity to monitories the noise transmission paths in the model [16].

\section{Overview of relevant literatures}

In the vehicle industry, there are lot of examples on employing SEA as well as the hybrid FE-SEA methods to vehicles. Typically, the investigation of a fully trimmed configuration requires lot of parameters about the structure itself and the poroelastic trim materials, which are defined in the form of Biot-parameters. Therefore, some authors investigated individual parts of vehicle only. The simulation of a passenger vehicle is usually made via hybrid FE-SEA method, while for some larger vehicles, such as a truck, classical SEA is typically sufficient. The following papers review the usage of hybrid FE-SEA methods for Trimmed Body as well as Body-In-White type vehicles.

\subsection{Literatures of Statistical Energy Analysis method}

Some of the papers in the literature deal with the development of the method itself [20], [25], mainly with improvements of the SEA as a numerical method. The most important parameters in experimental Statistical Energy Analysis are a) the determination of the CLFs and DLFs, b) the proper modelling techniques, i.e. which representation gives the best results for a panel?

Delaere et al. [17] investigated the air gap of an electric motor in 1999 via SEA. The focus was on the stator, so the rotor and the end caps were removed. Two different cases were considered: a) stator without coils, b) stator with standard coil system. The SEA used the experimental data in order to quantify the internal losses in the stator and the coils. This paper is interesting because electric machines rotate at high frequency, and this frequency is too high to fulfil the assumptions of the classical modal analysis techniques. Deleare et al. used inverse SEA to determine the internal energy flow as well as the system properties. The article described the operating condition of the machine, in which the stator teeth were excited by radial reluctant forces caused by the magnetic field in the air gap (Maxwell stress). The teeth transmitted these forces to the yoke without losses. The authors used Power Injection Method (PIM), in which every subsystem is excited, and the response is analysed in every case. Then, the Loss Matrices were calculated from the equations and the subsystems were covered randomly with $N$ accelerometers. The excitation was introduced at a random number of locations, using a shaker or hammer. The analysis concentrated on characterizing the internal loss factors and CLFs. 
Fischer's paper on SEA theory from 2006 [6] shown in the mathematic derivation of the kinetic energy, that this is proportional to the mean square velocity. Three averaging methods were introduced. The first one was over the points of the observation, while the second one over the points of excitation, where the information about the shape of the individual eigenmodes was eliminated. Note that as a consequence, the mode shape needs to be no longer considered in SEA. The third averaging was over the frequency of excitation. The author presented a really good analogy of the power balance equations through hydrodynamics. This article is a standard work on SEA, which helps to understand the theory and key aspects of the method.

Sarradj in 2004 [18] listed the most notable advantages of energy-based methods. According to him, energy-based methods are not sensitive to small parameter changes, since energy quantities can be averaged more easily. The goal of the energy-based computations is to determine the energy quantities (SPL). Sarradj described the SEA background and its basics, followed by an example, where the 1st and 4th subsystems were not coupled to each other, so their CLFs were zero. However, in the loss matrix all CLFs associated with power losses were summed. The author introduced two types of SEA, experimental and predictive, which can predict the behaviour of the structure in the early stages of design, when no object is available for measurements. The article highlights the main advantage of the SEA, that is that it can quantify the transmission paths in the system.

Hauer et al. [19] wrote an article about the hybrid approach for a trimmed passenger vehicle in 2004. The hybrid method was used to identify the loss factors within and between the subsystems, by using a Power Injection Method. According the authors, the hybrid method could calculate the tunnelling effect accurately, which means the physically not connected subsystems appeared as though coupled to each other. They investigated how many response data were necessary in order to obtain reliable results. For internal loss factors, 5 response points were enough, while for CLFs 10 points were required. As a conclusion, the authors stated that the hybrid method was a powerful method, especially in the early stages of development, since it allowed to determine accurately the SEA model parameters for complex systems and complex junctions. Another advantage of the hybrid method was that the optimization process used only two subsystems for determining the loss factors.

Different junction types can have a high impact on the Coupling Loss Factors. Panuszka, et al. [20] examined this effect in 2005 by investigating the Coupling Loss Factors between two rectangular plates joined either by a welded line, spot-weld, screw-bolted or riveted junctions. For the line junction, the authors investigated the influence of the thickness ratio of plates. For the point junction, the influence of the point distribution at the junctions was investigated. In the article, the equations of 
the junctions were derived. The test case was carried out with care, and the Internal Loss Factor of the plates was measured. The measurements were compared to theoretical equations and in some cases to simulation results as well. The results showed that the CLF values decrease when the ratio of the plate thicknesses is increased, as well as that the point-like junction has the tendency to increase with the density of the joining points. The highest CLF values appeared to spot welds, whereas the lowest to riveted joints. (See Fig. 4.)

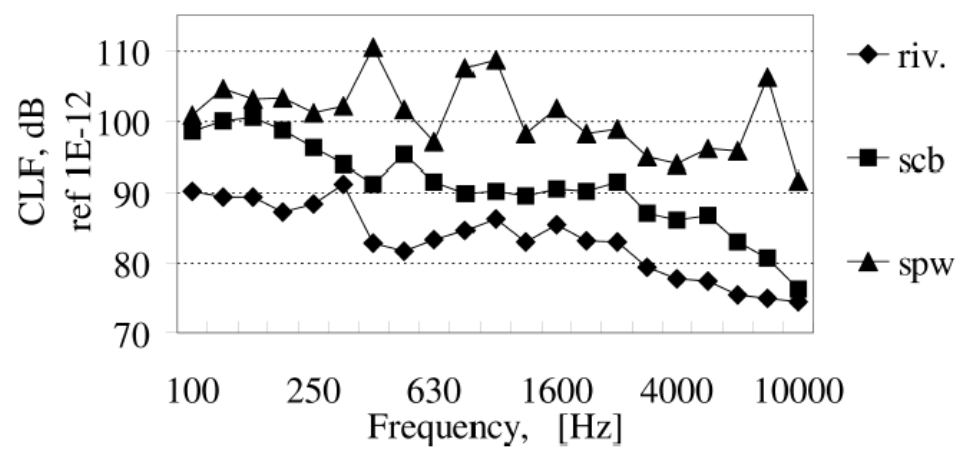

Figure 4. CLF results of different connection types versus the frequency [20]

In 2012, Xin et al. [21] investigated a car interior with SEA, for a vehicle at high speed, where air-borne excitation was the main contributor to interior SPL. They investigated a highly detailed model in SEA, with proper excitations. The acceleration of the engine mounts, and the SPL in the engine compartment were measured at $100 \mathrm{~km} / \mathrm{h}$. The air-borne excitation was obtained from CFD simulations, with high attention to the A-pillar and mirror area. The SPL at the driver's headspace was investigated from $200 \mathrm{~Hz}$ to $5 \mathrm{kHz}$. The comparison showed that the average deviation from the measurements was less than $3 \mathrm{~dB}$ (Fig. 5). 


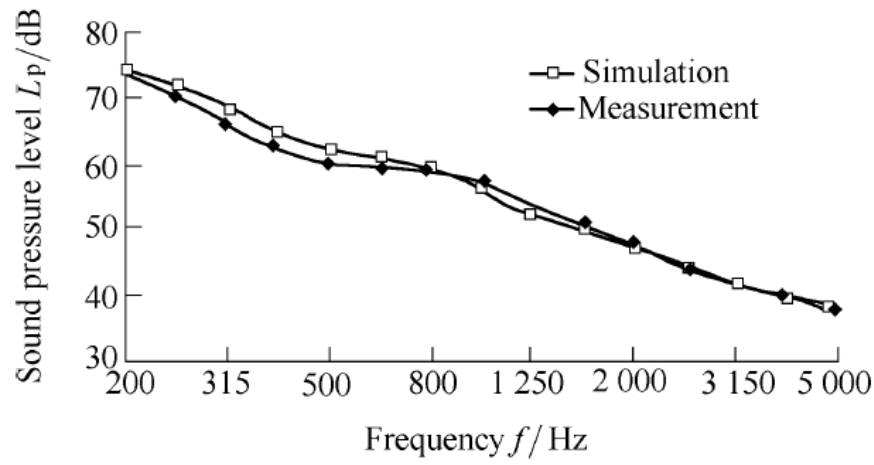

Figure 5. Compariosn of SPL results for an SEA simulation of a fully trimmed vehicle [21] Airborne noise excitation was obtained via CFD

Another objective of the work was to reduce the SPL in the driver headspace. The authors used a material database (AMDV) in order to set up different materials without measurements. There were 9 different load cases, and the results showed that they could reduce the SPL on average by $2,64 \mathrm{~dB}$, except at idling condition where the SPL increased by $2,2 \mathrm{~dB}$ [21].

Blanchet and Golota [22] have tried to answer in 2014 the following question: how can one identify the convective and acoustic sources as well as how can the transfer path from the sources to the interior cavity be identified? They used CFD and/or experiment to determine the turbulent flow, so that wind noise sources can be characterized. The investigation focused on the neighbourhood of the A-pillar as well as on the pressure fluctuations around the mirror area. They simulated three independent cavities, which one side was covered by glass and they examined different acoustic models (Corcos model, Diffuse Acoustic Field, Propagation Wavefield). The radiated acoustic power from the side glass was compared to SEA as well as Boundary Element Method (BEM), the results were found to compare quite well. During the wind tunnel test, the average SPL on the front left side glass and also in the interior were measured. When compared to the experiment, good agreement was achieved with SEA in a wide range of frequencies $(250 \mathrm{~Hz}-8 \mathrm{kHz})$, by using multiple propagating waves.

Putra et al. [23] worked out an individual method in 2014 to ensure the diffuse field for the SEA in a vehicle interior. They proposed the total energy to be equal to the direct field and reverberant field energies and removed the direct field component from the measurement and modified the DLFs and CLFs values. Fig. 6 shows the background of the analysis. 

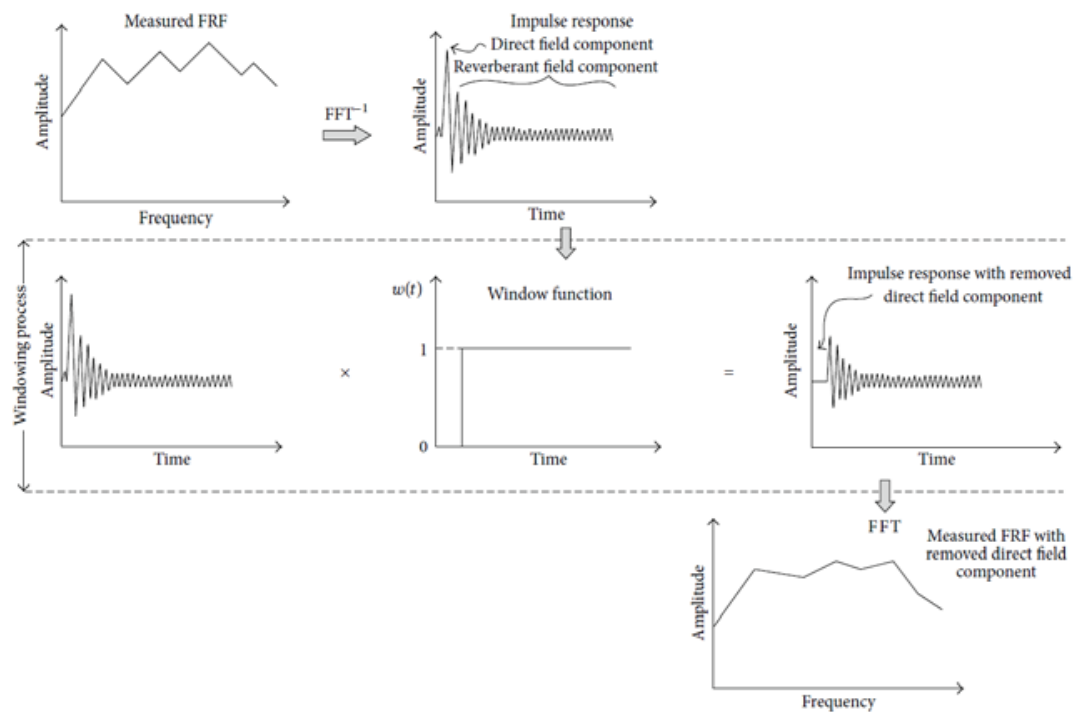

Figure 6. The direct field removing process [23]

They divided the vehicle interior into two subsystems, front and rear cabin. The front seats were the coupling between the cavities. During the measurements, a loudspeaker was used as an excitation, to inject the sound energy into the subsystem. Two cases were investigated: a) excitation from the loudspeaker, b) energy input from the engine at constant RPM. Sound intensity was measured, and the results compared to the classical SEA and to the corrected SEA methods. They tested a midsize vehicle in a semi-anechoic room. The corrected SEA simulations showed good correlation with the measurements, while the classical SEA overestimated the results at 1000, 2000 and 3000 RPM, as illustrated in Fig. 7. [23]. 


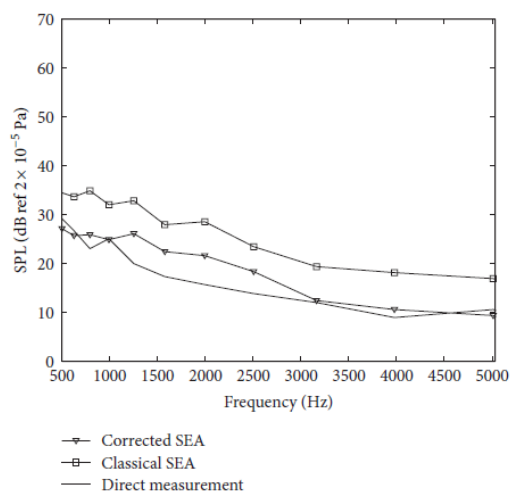

(a)

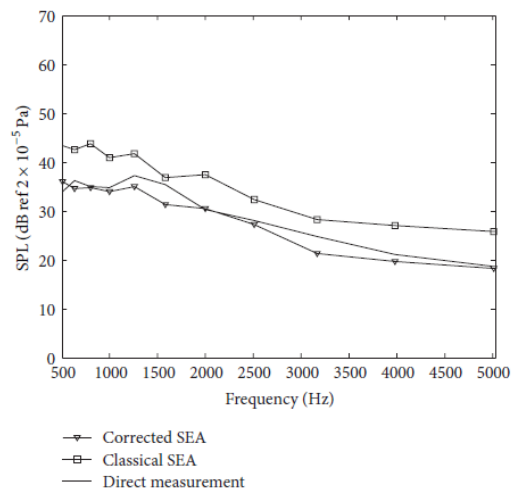

(b)

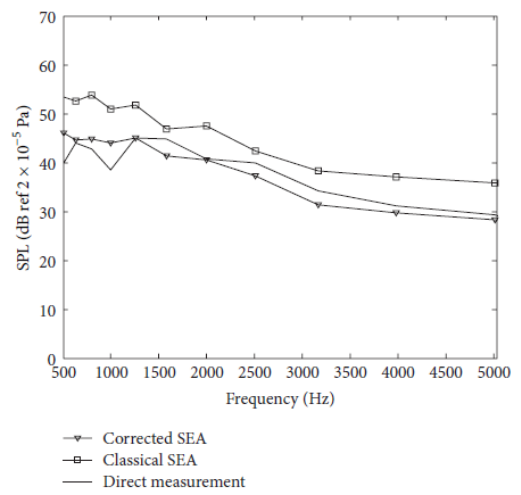

(c)

Figure 7. Results of classical and corrected SEA simualtions at the three engine RPMs [23]

With the advent of electric drive systems, the development of electric motors become important from fatigue and vibration point of view. Some papers investigated the problem with SEA only.

A. Bötke et al. [24] investigated in 2015 a fully trimmed electric car in SEA. They focused on the mid- and high-frequency range because of the high-frequency excitation. The road and the wind noise were the most dominant excitation sources, which were modelled as random signals. The authors described the limitations of FEM and explained why SEA is so powerful for these high-frequency analyses. According to Bötke et al., in case of electric cars, the location of the excitations is random, so it is required to analyse the whole vehicle and the whole sound insulation package, which increases the model size and computational costs in FEM. For the 
simulation model, various materials were experimentally characterized in terms of the poro-elastic properties (porosity, damping loss factor, etc.) Two different test cases were performed: a) a controlled environment in a semi-anechoic chamber, with a constant spherical sound source between $100 \mathrm{~Hz}-10 \mathrm{kHz}$ in the front and rear tire regions, using two different output nozzles; b) closed track tests at three constant speeds $(10,20,40 \mathrm{~km} / \mathrm{h})$. The measurements were performed at the driver head and the rear seat head positions with microphones. The results showed that differences of up to $10 \mathrm{~dB}$ could occur between the measurements and simulations. They listed the possible causes of errors, divided them into dependent and independent from the computation.

Gu and Sheng [25] developed a method in 2015 to estimate the CLFs from a structure coupled in series. They used the so-called Energy Ratio Method (ERM). In some cases, however, the original method had numerical problems, so they improved it by identifying a complex coupled system as two coupled systems. The improved method simplified the large matrix into a two-dimensional matrix. The classical ERM can cause negative CLF values, which is inconsistent. The objective of the paper was to substitute a structure consisting of $n$-coupled series by a two-coupled structure, as well as to substitute the loss factors and equivalent vibration energy of the new subsystems 1 and 2. In the experiment, they investigated the loss factors for each subsystem, when only one subsystem was excited. The results showed that there is no negative value in the improved method, compared to the classical ERM, which gave negative results in some frequency bands. Validation with experiments was performed by comparing the vibrational energies with simulations as well as tests of subsystems 1 and 2 . The prediction results appeared to be accurate and reliable.

Jang et al. [26] investigated in 2015 a mid-size truck in VA One and compared the results to measurements. They divided the truck into structural panel subsystems and acoustic subsystems, keeping in mind that the SEA subsystems must have at least 3 modes/bands in order to be valid. They considered the ribbing on the plate and the air duct in the pillars. For Noise Control Treatment definition, Biot parameters were applied. The TL for the grommets were specified analytically. If a panel was not an idealized SEA panel, they calculated it by a local FE model. Measurements were made on the road and on a 4-wheel dynamometer. The interior noise level was measured, and the structural and airborne transfer paths were investigated. According to the authors, in this case the air-borne sources could be neglected below $200 \mathrm{~Hz}$, while the structure-borne noise over $2 \mathrm{kHz}$. During the measurements, sound sources were placed around the vehicle and the SPL was measured at the driver and passenger headspaces. Tests were performed at multiple conditions: idle, wide open throttle for multiple gears, at constant $60,80 \mathrm{~km} / \mathrm{h}$, as well as at steady-state 
conditions. The conclusion was that the results correlated well, especially from 300 $\mathrm{Hz}$.

Jang et al. [27] analysed in 2015 the air-borne noise transfer path with SEA of a truck. They optimized the sound package of the vehicle, using multi-layer poroelastic materials. They identified the Biot-parameters of these by using an impedance tube and FOAM-X. The authors had two objectives: a) to improve the SPL at the driver head space at constant cost, b) to reduce the sound package cost at constant SPL. VA One has a Design Optimization Tool part, which was used to optimize the performance and the cost of the sound package. They measured the truck noise performance before and after the improved sound package implementation. For the beaded panels they used modal correction factors to amend the modal densities. The truck was tested in a semi-anechoic room. In this analysis, the structure-borne sources were not considered, only the air-borne sources. The measurement was performed at $80 \mathrm{~km} / \mathrm{h}$ at steady-state condition. Results showed that the sound package was enhanced by $3 \mathrm{~dB}$ at no additional cost, and that the price of the sound package was reduced by $30 \%$ at same SPL.

Jálics [28] wrote a short paper in 2017 about poroelastic materials and their possible mathematical descriptions in SEA. The intention was to determine the Transmission Loss of a plate in SEA through different models and to the compare them to a measurement. The plate was constrained between two cavities and the transmission loss was measured. There was a spherical speaker in the transmitter room, which provided the diffuse field, as well as some microphones. During the measurement, the reverberant time $\left(T_{60}\right)$ was measured in the receiver room. The SEA plate was modeled in three different ways, a) sandwich plate, b) composite plate, c) general multilayered plate. The results showed that the composite and multilayered plates had good arrangement in the frequency range of $200 \mathrm{~Hz}-2 \mathrm{kHz}$. Over $2 \mathrm{kHz}$, the sandwich plate had the best agreement with the measurements. The conclusion was that the difference was due to the damping value. Since this is unknown for such a wide frequency range, the author assumed a constant value.

In 2017, Siemens AG has issued a white paper on the challenges of vibroacoustic analysis of electric vehicles [1]. Electric motors have higher excitation frequencies than internal combustion engines and the accessories have higher impact to the interior noise, especially at idle and low speed. Road noise and wind noise is more prominent and thus they have greater impact on interior SPL. The problem with these excitation sources is that they are random and occur all around the car (Fig. 8). This implies the need for a full vehicle model with all noise control treatment. The size of such model is excessive for a classical FE model. Thus, SEA simulations offer a big advantage over FEM models in a sense that they are better suited for high frequency excitations and large models. The other issue with electric vehicles is that 
due to the large battery weight, weight reductions become more important. In order to design a lighter vehicle, lot of new materials were developed, which modelling is another new challenge in vibro-acoustics.

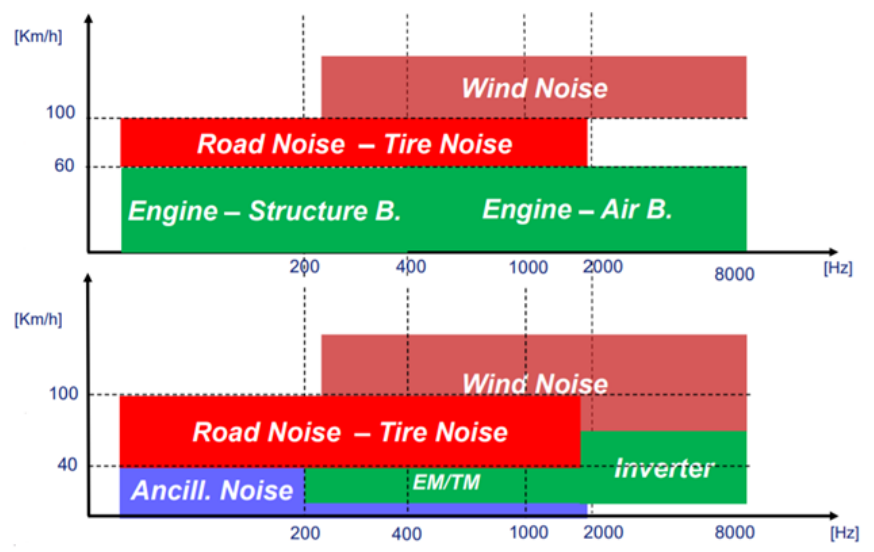

Figure 8. Contribution to interior noise for IC Engine driven vehicles (top), and Battery Electric Vehicles (bottom) [1]

An additional advantage of SEA for electric vehicles is that the modelling of exterior, infinite cavities is easier in SEA, so is the transfer path analysis as well (Fig. 9) [1].

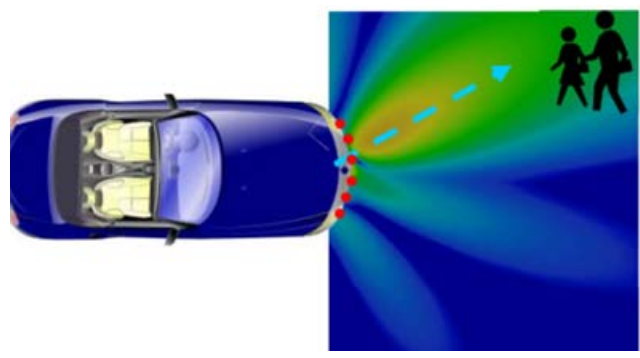

Figure 9. Exterior cavities have higher importance for electric vehicle [1]

\subsection{Literatures of Hybrid FE-SEA method}

Siano, et al. [29] have made an investigation of an engine cover. The authors used the hybrid FE-SEA method in different aspects. The geometry was modelled in FEM, while the cavity in SEA. The engine cover modelled as an FE model with very fine mesh, according to the authors it was able to perform the modal analysis with 
$100 \%$ accuracy up to $5 \mathrm{kHz}$. They also calculated transmission loss values of the cover, but the results were not compared to measurement results.

Charpentier et al. in 2007 [30] used hybrid FE-SEA to predict the structure-borne noise transmission in a trimmed automotive vehicle in the mid frequency range. The goal was to improve the SEA definition of the panels as well as the couplings used by the local FE models. They identified that simple structural junctions (welds, bolts) can be accurately estimated using the standard algorithms. The CLFs were described via the FE model. During the experiments, they applied huge number of accelerometers, typically 10 per/subsystem. Overall, 135 accelerometers were used for 15 load cases. Simulation and experimental results were found to be within $3 \mathrm{~dB}$ in most of the frequency bands.

Charpentier and Fukui [16] made a really detailed and advanced fully trimmed vehicle simulation by Hybrid FE-SEA in 2008. They investigated the mid-frequency domain between $200 \mathrm{~Hz}-1 \mathrm{kHz}$. They divided the experiment to different levels: first, a subsystem analysis, next a transmission path analysis (TPA) on the rails of the chassis. In order to reduce the computational costs, they compared the different FE models with the Hybrid FE-SEA model. The authors used different excitation points and different receiver points during the investigation, and they took into account the seals, passthroughs and leakage of the vehicle. The model was able to calculate the acceleration correctly far from the excitation point during the TPA. These time-consuming tests were successful according to the authors and the results were within $2 \mathrm{~dB}$. The instrument panel simulation times reduced from 20 hours to 7 hours. The difference between the models in terms of the interior noise was about 3-5 dB.

The usage of FE-SEA method in the mid-frequency range was the focus of Chen in 2011 [31] (Fig. 10). The author made an intensive full vehicle investigation. The stiff parts of the car were modelled in FE while the flexible parts as SEA panels. The effect of the trim parts was considered as an absorption coefficient. Comparison was made at $120 \mathrm{~km} / \mathrm{h}$, the maximum absolute error was less than $3 \mathrm{~dB}(\mathrm{~A})$. 


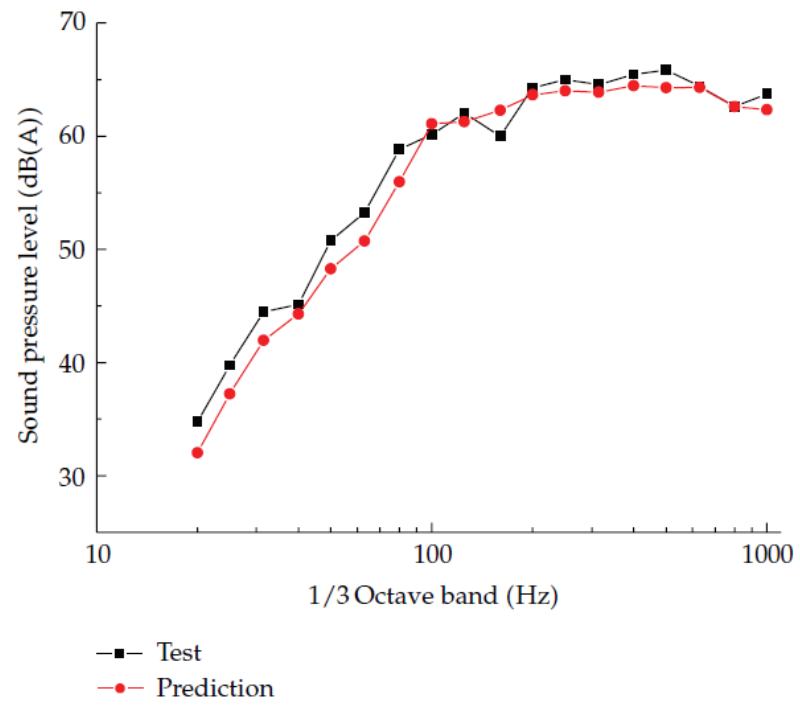

Figure 10. Simulation and measurement results [31] for a hybrid FE-SEA method applied to a fully-trimmed vehicle No information from the trim material properties was used during the prediction

Beigmoradi et al. [32] has performed an FE-SEA analysis of a car door in 2013. They made a clearance analysis of a B segment car door structure in order to improve the noise level in the driver's ear position. The trimmed door involved two parts: a trim panel and a pocket section. The clearance ranged between 0.1 and $6 \mathrm{~mm}$, and they investigated only the worst-case scenario nodes. As a result, they improved the rattle by adding ribs or by increasing the number of connection points. Adding some ribs on the pocket increased the stiffness of the region and reduced the rattle.

Prasanth et al. [15] made a really detailed hybrid FE-SEA investigation for a trimmed full car in 2011 with the aim to reduce structure borne noise. The authors focused on the mid-frequency range, between $200-1000 \mathrm{~Hz}$ and used Noise Transfer Path Analysis (NTPA) to identify the main contributors of the interior noise. The receiver cavities were at the driver's headspace and the right-rear seat headspace. Thanks to NTPA and the detailed car model, they could redesign the parts and observe the changes. For example, a modified windshield seal reduced the vibration on the windshield which was one of the main contributors to noise. Furthermore, the headliner modification reduced the SPL too, mainly in the rear seat region. The floor and the firewall were not critical parts in this analysis. The prediction was compared 
to measurement and according to the article, the biggest challenge was the repeatability of the cabin noise measurement. The SPL accuracy was $\pm 1 \mathrm{~dB}$. The model was able to predict the input power within $5 \mathrm{~dB}$ in most of the frequency bands.

Prasanth et al. [33] used hybrid FE-SEA method in 2013 to solve the midfrequency problem for a fully trimmed vehicle. The stiff parts were set up as an FE model, the panel-like structures as SEA model. They used multiple sources at different locations. The sound package was modelled using a classical SEA Transfer Matrix Method and local FE models were used for each subsystem to calculate the SEA parameters. For the SEA validation, accelerometers at 40 subsystems were used, while the number of total measurement locations was 300 . For the acoustic response, 40 microphones in 10 sub-cavities were applied to measure the SPL. The average difference was 3-5 $\mathrm{dB}$ between the measurement and the prediction. The simulation time was optimized and analysed in order to get the most accurate and reliable results as fast as possible. The fully trimmed model simulation took around 80 minutes on a regular PC.

A critical point, i.e. the definition of the correct Transmission Loss value between two cavities was addressed by $\mathrm{Wu}$ et al. in 2014 [34]. They developed a new approach to improve the Transmission Loss (TL) definition called Edge-based Smoothing FE-SEA (ES-FE-SEA). The goal was to make the stiff part of the deterministic model softer as well as to eliminate the numerical error in standard FEM. They compared the ES-FE-SEA results to a very smooth meshed FE model and a rough FE model. The second case was a dash panel and the results showed good agreement with the reference results. Also, computational efficiency was better than the reference one. The ES-FE-SEA method provided more accurate results than the traditional FE-SEA using the same mesh.

Tanner et al. [35] investigated in 2016 a vehicle floor that was excited between 0 - $3 \mathrm{kHz}$ frequency. A hybrid FE-SEA method was used to solve the mid-frequency gap. The stiff beams were modelled by FE, while the panels as SEA models. A new alternative model for the SEA subsystems called Discrete Flow Mapping (DFM) was also developed. The DFM result was compared to Monte Carlo FEM results, and it showed good agreement with the FEM results.

Kurosawa [36] used hybrid SEA in 2016 to predict the SPL in a vehicle interior. His model consisted of 106 structural subsystems, of which 10 served as input subsystems. The number of acoustic cavity subsystems was 30 . This model included the trims, damping materials, soundproof materials, as well as seals and leakages. Kurosawa determined the flow resistivity by Delany-Bazley's formula. The author pointed out that the flow resistivity will change if density changes. The sound absorption and sound insulation also changes. In conventional SEA model, the trim's 
thickness distribution is considered, and he improved it further by completing it with the density changes of the material. Measurements were carried out in a semianechoic room. The vehicle was measured on a chassis dynamo (CDM) as well as a test course (TC) in steady-state conditions. The difference between the two conditions was the air-borne excitation. The results of the comparison were that the SPL difference was $+-2 \mathrm{~dB}$ in the $800 \mathrm{~Hz}-5 \mathrm{kHz}$ frequency range. This paper is one of the most comprehensive ones, including a very detailed vehicle model, as well as detailed description.

\section{Conclusions}

This paper emphasized the importance of SEA methods as well as of Hybrid FESEA methods for the mid-frequency range vibroacoustic analyses. The literature review focused on the vehicle application and the cornerstones of the two methods. For example, the SEA method requires proper Coupling Loss Factors as well as Damping Loss Factors, etc. Some papers represent good and quite accurate simulation results in the mid-frequency range. Other sources present detailed vehicle models, although even in these papers the authors do not detail important model properties, such as DLFs or trim properties. Other important observation is that none of the literature described the method of how simulation from statistical methods and measurements shall be compared and the same is true for the comparison of two different simulation methods, such as the deterministic FEM and the statistically based SEA. This is expected to be a key element for objectively evaluating the accuracy of an SEA-based method, because some software use a closed formula to identify the power input or velocity response. All in all, there appears to be no generally accepted set simulation method that can achieve good correlation to measurements in the frequency range of $400 \mathrm{~Hz}-1 \mathrm{kHz}$.

\section{Acknowledgements}

Present work was supported by the MTA-SZE Lendület Vehicle Acoustics program as well as the EFOP 3.6.1-16-2016-00017 project.

\section{References}

[1] Siemens, Vibro-Acoustic Engineering Challenges in (Hybrid and) Electric Vehicles, Siemens AG, 2017.

[2] W. Desmet, B. Pluymers, O. Atak, "MID-FREQUENCY" CAE Methodologies for Mid-Frequency Analysis in Vibration and Acoustics, 2012, pp. 233-262. 
[3] P.J. Shorter, R.S. Langley, Vibro-acoustic analysis of complex systems, Journal of Sound and Vibration 288 (3) (2005) pp. 669-699. doi: $\underline{10.1016 / j . j s v .2005 .07 .010}$

[4] R. H. Lyon, R. G. DeJong, Theory and Application of Statistical Energy Analysis, 2nd Edition, Butterworth-Heinemann, Newton, MA, 1994.

[5] A. Le Bot, Foundation Statistical Energy Analysis in Vibroacoustics, 1st Edition, Oxford University Press, 2015.

[6] M. Fischer, Statistical Energy Analysis, Seminar: Vibrations and SturctureBorne Sound in Civil Engineering - Theory and Applications, 01. April, 2006

[7] N.J. Kessissoglou, J. Keir, A Hybrid Approach to Predict the Vibration Transmission in Ship Structures Using a Waveguide Method and Statistical Energy Analysis, Acoustics 2004, 3-5 November 2004, Gold Coast, Australia, pp. 587-592.

[8] B. Campolina, Vibroacoustic modelling of aircraft double-walls with structural links using Statistical Energy Analysis (SEA), Acoustics [physics.class-ph]. Université de Sherbrooke; Université Pierre et Marie Curie - Paris VI, 2012. English. <tel-00736619>

[9] H. S. Malushte, Evaluation of Statistical Energy Analysis for Prediction of Breakout Noise from Air Duct, Architectural Engineering, University of Nebraska, Lincoln, Spring 4-19-2013

[10] I. Dokhani, N.V.H. Analysis of a Vehicle using Statistical Energy Analysis, Dissertation, Blekinge Institute of Technology, Karlskrona, Sweden (2014).

[11] Lyon, R. H., Maidanik, G., Power Flow Between Linearly Coupled Oscillators, Journal of the Acoustical Society of America 34 (623) (1962) pp. 623-639.

doi: https://doi.org/10.1121/1.1918177

[12] A. Le Bot, N. Totaro, T. Lafont, Statistical Energy Analysis, Assumptions and Validity, Medyna 2017: 2nd Euro-Mediterranean Conference on Structural Dynamics and Vibroacoutics, Sevilla, Spain, 25-27 Apr. 2017. 
[13] D. Johansson, P. Comnell, Statistical Energy Analysis software; Development and implementation of an open source code in Matlab/Octave, Master's Thesis, Chalmers University of Technology, Göteborg, Sweden (2010).

[14] W. Desmet, Mid-frequency vibro-acoustic modelling: Challenges and potential solutions, Proceeding of ISMA2002 - Volume II, Katholieke Universiteit Leuven Department of Mechanical Engineering, Heverlee, Belgium, 2002, pp. 835-862.

[15] S. Prasanth, A. Charpentier, K. Fukui, Using the Hybrid FE-SEA Model of a Trimmed Full Vehicle to Reduce Structure Borne Noise from $200 \mathrm{~Hz}$ to $1 \mathrm{kHz}$, SAE Technical Paper 2011-26-0020, 2011. doi: https://doi.org/10.4271/2011-26-0020

[16] A. Charpentier, P. Shreedhar et al., Modeling process and validation of Hybrid FE-SEA method to structure-borne noise paths in a trimmed automotive vehicle, 2008 SAE Brasil Noise and Vibration Conference, Florianópolis, SC, Brazil, March 30th to April 01st, 2008. doi: https://doi.org/10.4271/2008-36-0574

[17] K. Delaere, M. Iadevaia et al., Statistical Energy Analysis of Acoustic Noise and Vibration for Electric Motors: Transmission from Air gap Field to Motor Frame, Conference Record of the 1999 IEEE Industry Applications Conference, Thirty-Forth IAS Annual Meeting, Phoenix, AZ, USA, 1999, pp. 1897-1902.

doi: https://doi.org/10.1109/IAS.1999.805997

[18] E. Sarradj, Energy-based vibroacoustics: SEA and beyond, Proceedings of the Joint Congress CFA/DAGA'04, Strasbourg, France, 2004, pp. 1157-1162.

[19] I. Hauer, K. Jalics et al., Prediction of Vehicle Interior Noise in High Frequency Range using Statistical Energy Analysis Hybrid Method, Proceedings of the Joint Congress CFA/DAGA'04, Strasbourg, France, 2004, pp. 11-12.

[20] R. Panuszka, J. Wiciak, M. Iwaniec, Experimental Assessment of Coupling Loss Factors of Thin Rectangular Plates, Archives of Acoustics 30 (4) (2005) pp. 533-551. 
[21] C. Xin, D. Wang, Z. Ma, Simulation on a Car Interior Aerodynamic Noise Control Based on Statistical Energy Analysis, Chinese Journal of Mechanical Engineering 25 (5) (2012) pp. 1016-1021.

[22] D. Blanchet, A. Golota, Validation of a wind noise source characterization method for vehicle interior noise prediction, Proceedings of ISMA 2014 International Conference on Noise and Vibration Engineering, USD 2014 International Conference on Uncertainty in Structural Dynamics, Leuven, 2014, pp. 241-256.

[23] A. Putra, A. Munawir, W.M. Farid, Corrected Statistical Energy Analysis Model for Car Interior Noise. Advances in Mechanical Engineering (2015) doi: https://doi.org/10.1155/2014/304283

[24] A. Bötke, E. Erensoy, C. Sevginer, Modeling and Validation Processes of an Electric Vehicle with Statistical Energy Analysis, Euronoise 2015, Maastricht, 2015, pp. 1417-1422.

[25] J. Gu, M. Sheng, Improved Energy Ratio Method to Estimate Coupling Loss Factors for Series Coupled Structure, Journal of Mechanical Engineering 45 (1) (2015) pp. 37-40.

doi: https://doi.org/10.3329/jme.v45i1.24382

[26] Y. S. Jang, J. Y. Kuk et al., SEA Modeling and Validation of a Truck Cab for Sound Package Optimization, Inter.Noise Conference 2015, San Francisco, California, USA, 2015, p. 12.

[27] Y. S. Jang, J. Y. Kuk et al., Optimization of the Sound Package of a Truck using Statistical Energy Analysis, Inter.Noise Conference 2015, San Francisco, California, USA, 2015, p. 12.

[28] J. Károly, Investigation of Acoustic Properties of Poroelactic Materials with Measurement and Simulation, GÉP 68 (4) (2017) pp. 11-14, in Hungarian

[29] D. Siano, M. Viscardi, R. Aiello, An Hybrid FE/SEA Approach for Engine Cover Noise Assessment, Recent Researches in Mechanical and Transportation Systems, pp. 199-205. 
[30] A. Charpentier, P. Shreedhar, K. Fukui, Using the Hybrid FE-SEA Method to Predict Structure-Borne Noise Transmission in a Trimmed Automotive Vehicle, SAE Technical Paper 2007-01-2181, 2007.

doi: https://doi.org/10.4271/2007-01-2181

[31] S.M. Chen, D.F. Wang, J.M. Zan, Interior Noise Prediction of the Automobile Based on Hybrid FE-SEA Method, Mathematical Problems in Engineering, 2011 (Article ID 327170) p. 20. doi: https://doi.org/10.1155/2011/327170

[32] S. Beigmoradi, K. Jahani, H. Hajabdollahi, Squeak and rattle noise prediction for trimmed door of a car using hybrid statistical energy - Finite element method analysis, The Journal of the Acoustical Society of America 133 (5) (2013) pp. 3462-3462.

[33] S. Prasanth, P. Shreedhar et al., Modeling process and validation of Hybrid FE-SEA method to structure-borne noise paths in a trimmed automotive vehicle, International Journal of Research in Aeronautical and Mechanical Engineering 1 (3) (2013) pp. 17-28.

[34] F. Wu, G.R. Liu et al., Mid-frequency prediction of transmission loss using a novel hybrid deterministic and statistical method, JVE International LTD. Vibroengineering Procedia 4 (2014) pp. 34-39.

[35] G. Tanner, D.J. Chappell, Comparison of Methods for Modelling Mid-toHigh Frequency Vibro-Acoustic Energy Distributions in a Vehicle Floor Structure, SAE International 2016, 06/15/2016 doi: https://doi.org/10.4271/2016-01-1853

[36] Y. Kurosawa, Predicting Automotive Interior Noise Including Wind Noise by Statistical Energy Analysis, International Journal of Mechanical and Mechatronics Engineering 10 (3) (2016) pp. 635-641. 